\title{
Determinantes para a prevalência de sífilis congênita em um hospital de clínicas
}

\author{
Determinants for the prevalence of congenital syphilis in a clinical hospital \\ Determinantes de la prevalencia de sífilis congénita en un hospital clínico
}

Recebido: 06/01/2022 | Revisado: 11/01/2022 | Aceito: 12/01/2022 | Publicado: 14/01/2022

\author{
Thayna Karolina Gomes Fernandes \\ ORCID: https://orcid.org/0000-0001-6864-2067 \\ Universidade Federal do Triângulo Mineiro, Brasil \\ E-mail: thaynakarolina_10@ @otmail.com \\ Jussara Silva Lima \\ ORCID: https://orcid.org/0000-0002-3010-9053 \\ Universidade Federal do Triângulo Mineiro, Brasil \\ E-mail: jussarasilvalima2014@gmail.com \\ Wellington Francisco Rodrigues \\ ORCID: https://orcid.org/0000-0002-3426-2186 \\ Universidade Federal do Triângulo Mineiro, Brasil \\ E-mail: wellington.frodrigues@ hotmail.com
}

\begin{abstract}
Resumo
Este estudo teve como objetivo relatar os casos de sífilis congênita (SC) e os seus principais fatores associados em uma região do Triângulo Mineiro, Brasil. Trata-se de uma pesquisa quantitativa e transversal realizada entre 2014 a junho de 2019. Os dados das crianças e das mães no que tange o pré-natal, parto, puerpério foram extraídos em prontuários de pacientes com SC em Hospital de Clínicas da Universidade Federal do Triângulo Mineiro no município de Uberaba. A coletada de dados foi guiada pelo Protocolo de Investigação de Casos de SC Precoce de 2014 do Ministério da Saúde. Foram encontrados 129 casos da doença e fatores como idade, grau de instrução, dependência química, cor/raça influenciaram em maior prevalência da doença. Além disso o tratamento materno foi inadequado em $93.75 \%$. A prevalência e as relações de SC observada provocam questionamentos sobre a qualidade equitativa da assistência pré-natal e perinatal ofertada à população.
\end{abstract}

Palavras-chave: Treponema pallidum; Sífilis congênita; Cuidado pré-natal; Saúde da mulher; Prevalência.

\begin{abstract}
This study aimed to report cases of congenital syphilis (CS) and its main associated factors in a region of the Triângulo Mineiro, Brazil. This is a quantitative and cross-sectional research carried out between 2014 and June 2019. Data from children and mothers regarding prenatal care, childbirth, puerperium were extracted from medical records of patients with CS at Hospital de Clínicas of the Federal University of the Triângulo Mineiro in the municipality of Uberaba. Data collection was guided by the Ministry of Health's 2014 Early CS Case Investigation Protocol. 129 cases of the disease were found and factors such as age, education level, chemical dependency, color/race influenced a higher prevalence of the disease. Furthermore, maternal treatment was inadequate in $93.75 \%$. The prevalence and relationships of CS observed raise questions about the equitable quality of prenatal and perinatal care offered to the population.
\end{abstract}

Keywords: Treponema pallidum; Congenital syphilis; Prenatal care; Women's health; Prevalence.

\section{Resumen}

Este estudio tuvo como objetivo reportar casos de sífilis congénita (SC) y sus principales factores asociados en una región del Triângulo Mineiro, Brasil. Se trata de una investigación cuantitativa y transversal realizada entre 2014 y junio de 2019. Se extrajeron datos de niños y madres sobre atención prenatal, parto, puerperio de las historias clínicas de pacientes con SC en el Hospital de Clínicas de la Universidad Federal del Triângulo Mineiro. en el municipio de Uberaba. La recopilación de datos se guió por el Protocolo de investigación de casos de CS temprana del Ministerio de Salud de 2014.Se encontraron 129 casos de la enfermedad y factores como la edad, el nivel educativo, la dependencia química, el color / raza influyeron en una mayor prevalencia de la enfermedad. Además, el tratamiento materno fue inadecuado en el $93,75 \%$. La prevalencia y las relaciones de SC observadas plantean interrogantes sobre la calidad equitativa de la atención prenatal y perinatal ofrecida a la población.

Palabras clave: Treponema pallidum; Sífilis congénita; Atención prenatal; Salud de la mujer; Prevalencia. 


\section{Introdução}

As infecções sexualmente transmissíveis (IST) são consideradas um problema crucial de saúde pública e estão entre as doenças transmissíveis mais prevalentes. Elas ocasionam danos socioeconômicos mundiais, com impacto direto na saúde da mulher e da criança (Bezerra et al, 2019; Monu et al, 2020). Dentre as ISTs, a sífilis merece notoriedade, visto que é uma doença totalmente passível de prevenção que vem apresentando infecção reemergente (Lafetá et al, 2016). A sífilis, causada pelo Treponema pallidum (TP), é uma doença infecciosa crônica, que acomete praticamente todos os órgãos e sistemas e pode ocasionar sequelas irreversíveis (Rowe et al, 2018; Koundanya \& Tripathy, 2021).

A sífilis congênita (SC) é proveniente da disseminação hematogênica da bactéria TP na gestante infectada e não tratada ou inadequadamente tratada para o feto por via placentária. A transmissão vertical ocorre principalmente intraútero, embora também possa ser transmitida durante o parto, a partir do contato do recém-nascido com lesões genitais materna. A infecção fetal é influenciada pelo estágio da sífilis na mãe e pela duração da exposição do feto, podendo ocorrer em qualquer fase da gestação, sendo a probabilidade de transmissão maior nas fases iniciais da doença materna, quando existe número elevado de espiroquetas no sangue (Rowe et al, 2018; Woods, 2005). A contaminação do concepto pode ocasionar abortamento espontâneo, parto prematuro, baixo peso ao nascimento, óbito fetal, morte neonatal e sequelas motoras, cognitivas, neurológicas, visuais e auditivas (Andrade et al 2018; Kroeger et al, 2018).

Apesar da prevalência da infecção pelo TP ter sido atenuada com a descoberta da penicilina, em 1943, a partir dos anos 60 e principalmente na década de 1980, houve uma tendência mundial de exacerbação da sífilis (De Lorenzi \& Madi, 2001). Na última década houve um aumento do número de casos de sífilis em gestantes, sífilis congênita e sífilis adquirida. No Brasil, segundo o Ministério da Saúde (MS), 50 mil parturientes têm o diagnóstico de sífilis por ano, com prevalência variando de 1,1 a 11,5\%, ou seja, aproximadamente 12 mil nascidos vivos têm SC no país a cada ano (Lafetá et al, 2016).

A SC é classificada em precoce quando as manifestações clínicas surgem nos dois primeiros anos de vida da criança e em tardia quando os sintomas aparecem após o segundo ano de vida (Andrade et al, 2018). Aproximadamente 50\% dos recémnascidos infectados são assintomáticos ao nascimento. As lesões de pele e das mucosas da SC precoce podem surgir desde o nascimento, sendo as mais frequentes, o exantema maculoso na face e extremidades, as lesões bolhosas, o condiloma latum, as fissuras periorais e anais, o pênfigo palmo-plantar e a rinite sero-sanguinolenta. Outros sintomas observados são hepatoesplenomegalia, icterícia, linfadenopatia, osteocondrite, periostite ou osteíte, anemia e hidropsia fetal. Na SC tardia, as lesões mais evidentes são fronte olímpica, palato em ogiva, nariz em sela, rágades periorais, tíbia em sabre, dentes de Hutchinson e molares em formato de amora, assim como ceratite, surdez e retardo mental (Forrestel et al, 2020; Avelleira \& Bottino, 2006).

A SC é um evento sentinela, uma vez que pode ser evitada por ações eficazes de saúde e obriga a investigação retrospectiva dos casos para se obter informações da assistência prestada e propor intervenções pertinentes (Domingues et al, 2013). As taxas de SC em uma determinada região podem favorecer a avaliação da atenção primária à saúde de cada município, uma vez que esta retrata a qualidade da assistência pré-natal de cada população (Bezerra et al, 2019).

O propósito de controle da sífilis é o bloqueio da transmissão em série e a prevenção de novos casos. A medida mais eficaz para evitar a transmissão da doença consiste na detecção e no tratamento precoce durante o pré-natal, já que a maioria dos casos acontece porque a gestante não foi testada para sífilis ou porque recebeu tratamento inadequado antes ou durante a gestação (Rose, 1998; Cooper \& Sánchez, 2018; Phiske, 2014).

Diante da relevância da SC no cenário da saúde pública mundial e da necessidade de se conhecer a população atendida em cada local, o presente estudo objetivou relatar os casos de SC e os seus principais fatores associados em uma região do Triângulo Mineiro, Brasil. 


\section{Metodologia}

Foi realizado um estudo observacional transversal retrospectivo, entre o período de 2014 a junho de 2019 (Rodrigues et al, 2016). Os dados foram obtidos em registros médicos em prontuários de pacientes com sífilis congênita em Hospital de Clínicas da Universidade Federal do Triângulo Mineiro, situado na região sudeste do Brasil, no Estado de Minas Gerais, município de Uberaba. A coletada de dados foi realizada no período de maio a agosto de 2020 da qual foi guiada pelo Protocolo de Investigação de Casos de Sífilis Congênita Precoce de 2014 do Ministério da Saúde.

Os dados contidos nos prontuários dos pacientes foram acessados e avaliados após consentimento dos setores responsáveis, Além disso para prevenir a perda de confidencialidade dos dados, que pode levar a exposição do paciente, o trabalho foi executado em conformidade as diretrizes de não maleficiência e de beneficência, a fim de assegurar os direitos dos participantes, em consonância as normativas de assistência integral; assim como, de respeitar os participantes da pesquisa em sua plena dignidade e autonomia. A pequisa seguiu os princípios éticos contidos na Declaração de Helsinque, bem como na Resolução 466/12 do Conselho Nacional de Saúde. Todos os procedimentos de pesquisa foram avaliados e aprovados pelo Comitê de Ética em Pesquisa do HC UFTM (número de aprovação: 3.318.756).

Casos de sífilis congênita em crianças internadas no Hospital de Clínicas no período de 2014 a junho de 2019 foram selecionados em prontuários físicos e eletrônicos. Informações das crianças e das mães no que tange o pré-natal, parto, puerpério foram extraídos. Os dados sobre a mãe como idade, estado civil, escolaridade, profissão, raça/cor, dependencia química, coinfecção, paridade, momento de diagnóstico de sífilis, número de consultas pré-natal, idade gestacional na primeira consulta de pré-natal, teste não treponêmico, realização ou não de tratamento adequado materno, estado clínico e sorológico do parceiro, presença ou ausência de tratamento do parceiro sexual, assim como dados sobre o recém-nascido como tipo do parto, peso ao nascimento, idade gestacional ao nascer, teste não treponêmico do sangue periférico e do líquor, sinais e sintomas clínicos, alterações no raio-x de ossos longos, realização ou não do tratamento adequando ao recém-nascido e presença ou ausência de seguimento clínico e sorológico da criança até os 18 meses foram considerados para avaliação. Dados inconclusiveis ou faltantes nos prontuários não foram incluídos para avaliação.

Os dados foram tabulados no programa Microsoft® Excel e analisados no IBM SPSS statistics 21 e GraphPad Prism 7.0. Para determinação das prevalências no munícipio foram considerados os dados do Instituto Brasileiro de Geográfia e Estatística. Os dados seguiram para análise descritiva relatada em frequências relativas e absolutas, assim como verificado a relação entre as frequências para algumas das variáveis. As hipóteses foram testadas pelos testes Qui-quadrado. O teste de Spearman foi utilizado para correlações não paramétricas. As diferenças observadas foram consideradas significativas quando $\mathrm{p}<0,05(5 \%)$ (Arango, 2001).

\section{Resultados}

Após a obtenção, tratamento e avaliação dos dados levantados foram encontrados 129 casos relacionados à sífilis congênita no período avaliado. Nem todos os casos atenderam ao preenchimento de todas as variáveis analisadas neste estudo. Inicialmente as prevalências dos casos de sífilis congênita em relação a diferentes faixa-etárias, escolaridade, número de gravidez, ter ou não dependência química e cor/raça foram avaliados (Tabela 1). As correções das frequências absolutas foram realizadas pelos dados censitários municipal e foi possível observar uma maior prevalência dos casos em mulheres de 20 a 30 anos (289,98 casos por 100 mil habitantes), chegando a quase 4 vezes a mais em relação à faixa etária dos 31 aos 40 anos (74,86 casos por 100 mil habitantes), seguido por 65 vezes a mais do que mulheres acima de 40 anos (4,43 casos por 100 mil habitantes).

A segunda prevalência mais frequente foi para a faixa etária menor que 20 anos (256,43 casos por 100 mil habitantes), com 3 vezes a mais do que a faixa etária dos 31 aos 40 anos e com prevalência 57 vezes maior do qua a faixa etária acima dos 
40 anos. Com os dados foi possível determinar uma correlação negativa significativa em relação as prevalências e as idades das mulheres envolvidas com sífilis congênita ( $r$ ho $=-0,95, p=0,03$ ).

Em relação a escolaridade foi possível observar uma maior frequência dos casos em mulheres com ensino fundamental ( $\mathrm{n}=74-60,16 \%)$ em relação às mulheres com ensino médio $(\mathrm{n}=49-39,84 \%)$, um efeito de 1,5 vezes a mais na frequência de associação com sífilis congênita em mulheres vinculadas ao ensino fundamental foi encontrado, não foi notificado nenhum caso associado às mulheres com ensino superior neste levantamento.

O número de gravidez não foi um fator associado às frequências de sífilis congênita ( $p>0,99)$, entretanto mulheres com 2 ou 3 gestações apresentaram uma frequência maior em 19,84 \% em relação às mulheres com apenas uma gestação e em 18,25\% em relação à mulheres com 4 ou mais gestações. A correlação pelo número de gestações e os casos de sífilis pode demonstrar uma fraca correlação positiva $($ rho $=0,50$ ), indicando a não relação significativa ou preditora do número de gestações e frequência de sífilis congênita.

A dependência química influenciou positivamente na frequência da doença com uma taxa de elevação da frequência dos casos em $8,51 \%$. Na avaliação das prevalências entre a categoria cor/raça foi identificada uma maior prevalência para mulheres negras em relação às brancas (aumento de 2,6 vezes) ou pardas (aumento de 2,44 vezes) (Tabela 1). 
Research, Society and Development, v. 11, n. 1, e54511125419, 2022

(CC BY 4.0) | ISSN 2525-3409 | DOI: http://dx.doi.org/10.33448/rsd-v11i1.25419

Tabela 1 - Principais fatores associados a mulheres relacionadas à sífilis congênita na região do Triângulo Mineiro, Sudeste do Brasil no período de 2014 a junho de 2019.

\begin{tabular}{|c|c|c|c|}
\hline Faixa etária & & & \\
\hline Intervalos & Casos - N & $\begin{array}{l}\text { Mulheres residentes - } \\
\mathrm{N}^{*}\end{array}$ & $\begin{array}{l}\text { Prevalência/ } 100 \text { mil } \\
\text { hab. }\end{array}$ \\
\hline$<20$ anos & 31 & 12089 & 256,43 \\
\hline 20-30 anos & 79 & 27243 & 289,98 \\
\hline $31-40$ anos & 18 & 24046 & 74,86 \\
\hline$>40$ anos & 1 & 22550 & 4,43 \\
\hline Total & 129 & 85928 & 625,71 \\
\hline $\mathrm{CV}-\%$ & 103,88 & 30,54 & 88,58 \\
\hline Spearman $\mathrm{r}$ & & & $-0,95$ \\
\hline Valor $\mathrm{p}$ & & & $0,03 *$ \\
\hline \multicolumn{4}{|c|}{ Escolaridade } \\
\hline Perfil & Casos - N & Frequência - \% & Relação (F/M) \\
\hline Ens. Fundamental & 74 & 60,16 & 1,51 \\
\hline Ens. Médio & 49 & 39,84 & \\
\hline Total & 123 & 100,00 & \\
\hline \multicolumn{4}{|c|}{ Gravidez } \\
\hline $\mathrm{N}$ & Ocorrências - N & $\begin{array}{l}\text { Casos/perfil de } \\
\text { gravidez - \% }\end{array}$ & Valor $\mathrm{p}$ \\
\hline 1 & 33 & 26,19 & \\
\hline 2 ou 3 & 58 & 46,03 & $>0,99$ \\
\hline 4 ou maior & 35 & 27,78 & \\
\hline Total & 126 & 100,00 & \\
\hline Spearman $\mathrm{r}$ & & & 0,50 \\
\hline \multicolumn{4}{|l|}{ Dependência química } \\
\hline Perfil & Casos/perfil - N & Frequência/perfil - \% & Relação (sim/não) \\
\hline Sim & 51 & 52,04 & 1,09 \\
\hline Não & 47 & 47,96 & \\
\hline Total & 98 & 100,00 & \\
\hline Taxa de aumento - $\%$ & & & 8,51 \\
\hline \multicolumn{4}{|c|}{ Cor/Raça } \\
\hline Descrição & Caso/característica - $\mathrm{N}$ & $\begin{array}{c}\text { Indivíduos/ } \\
\text { característica - } \mathrm{N} \\
\end{array}$ & Prevalência/ 100 mil** \\
\hline Branca - (a) & 45 & 4529 & 993,60 \\
\hline Negra - (b) & 45 & 1742 & 2583,24 \\
\hline Parda - (c) & 39 & 3690 & 1056,91 \\
\hline Total & 129 & 9961 & 4633,75 \\
\hline Relação b/a & & & 2,60 \\
\hline Relação b/c & & & 2,44 \\
\hline
\end{tabular}

*http://www.uberaba.mg.gov.br/portal/acervo/saude/arquivos/plano_municipal_saude.pdf. ** CENSO, I. B. G. E. Disponível em:< http://www. censo2010. ibge. gov. br/>. CV = coeficiente de variação. Hab = habitantes. Fonte: Dados da pesquisa (2021). 
Após descrever as principais associações sobre as prevalências da sífilis congênita e faixa-etárias, escolaridade, número de gravidez, ter ou não dependência química e cor/raça das mulheres relacionadas o estudo foi verificar possível relações vinculadas à coinfeções, número de consultas no pré-natal e ao tratamento materno adequado após laudo positivo para sífilis (Tabela 2). O laudo positivo para sífilis foi associado com uma diminuição de coinfecção em quase 87,40\% nas gestantes avaliadas, essa taxa foi relacionada com uma frequência maior de consultas em gestantes positivas para sífilis ( $\mathrm{n}=79-$ 65,83\%) em relação as negativas ( $\mathrm{n}=41-34,17 \%)$, com uma taxa de elevação na positividade de sífilis em 92,68\%. Por fim, em relação a adequação da terapia frente a doença a maioria dos relatos não foram satisfatórios $(n=90-93,75 \%)$ o que influenciou em uma relação de inadequação de 15 para 1 de tratamento adequado $(n=6-6,25 \%)$ (Tabela 2).

Tabela 2 - Influência da coinfeção, número de consultas no pré-natal e adequação no tratamento materno de mães vinculadas à sífilis congênita em região do Triângulo Mineiro, Sudeste do Brasil no período de 2014 a junho de 2019.

\begin{tabular}{|c|c|c|c|}
\hline Coinfecção & & & \\
\hline Perfil & Ocorrências - N & Casos/perfil de gravidez - $\%$ & Relação (não/sim) \\
\hline Não & 119 & 93,70 & \multirow{2}{*}{14,88} \\
\hline Sim & 8 & 6,30 & \\
\hline Total & 127 & 100,00 & \\
\hline Taxa de aumento - $\%$ & & & 1387,50 \\
\hline \multicolumn{4}{|c|}{ Consultas no pré-natal $-\mathrm{N} \geq 6$} \\
\hline Perfil & Casos/perfil - N & Frequência/perfil - \% & Relação (sim/não) \\
\hline $\mathrm{N} \geq 6$ & 79 & 65,83 & \multirow{2}{*}{1,93} \\
\hline $\mathrm{N}<6$ & 41 & 34,17 & \\
\hline Total & 120 & 100,00 & \\
\hline Taxa de aumento - $\%$ & & & 92,68 \\
\hline \multicolumn{4}{|c|}{ Tratamento materno adequado } \\
\hline Descrição & Ocorrências - N & Frequência/perfil - \% & Relação (não/sim) \\
\hline Não & 90 & 93,75 & \multirow{2}{*}{15,00} \\
\hline Sim & 6 & 6,25 & \\
\hline Total & 96 & 100 & \\
\hline Taxa de aumento - $\%$ & & & 1400,00 \\
\hline
\end{tabular}

Fonte: Dados da pesquisa (2021).

\section{Discussão}

A sífilis é uma doença frequente em países em desenvolvimento, incluindo o Brasil, representando um problema mundial de saúde pública (Galvis \& Arrieta, 2020). A SC permanece como uma doença a ser prevenida e tratada, sendo crucial identificar os fatores de risco que levam a transmissão vertical do TP (Cooper \& Sánchez, 2018). Aqui, no presente estudo foi possível encontrar na região avaliada 129 casos da doença em um período aproximado de 6 anos onde foram identificados fatores que influenciaram no aumento das frequências da doença, como idade, grau de instrução, dependência química, cor/raça. Além disso os dados demonstraram uma inadequação ao tratamento materno na maioria dos casos.

Os dados encontrados permitem corroborar com alguns fatores já observados em outros estudos associados ao aumento da frequência de SC, como predomínio da cor negra e parda, baixa escolaridade, idade de 20 a 29 anos, início da atividade sexual precoce e estado civil solteiro (Magalhães et al, 2013; Paiva et al, 2020). No entanto os fatores vinculados as 
taxas da doença parecem ser influenciados por aspectos regiões, haja vista que em outro estudo os autores relataram infecção pelo TP em mulheres com nível elevado de escolaridade e relação conjugal estável, com um único parceiro sexual (Almeida et al, 2009).

A adolescência é apontada como fator de risco importante para contrair ISTs, incluindo a sífilis (Santos et al, 2019). O perfil epidemiológico da doença tem sofrido algumas modificações, mas no que tange a faixa etária pode-se encontrar semelhanças da distribuição mesmo comparado a mais de uma década atrás, onde em um estudo publicado em 2006 relatam com dados oriundos da Maternidade da Santa Casa do Pará, que na ocasião que 26\% das mães com recém-nascidos com sífilis eram adolescentes (Araujo et al, 2006). No estudo citado anteriormente os autores observaram um predomínio de mulheres entre 20 e 30 anos de idade, solteiras, com baixa escolaridade, sendo a maioria parda e negra.

A identificação da gestante com sífilis durante o pré-natal é primordial uma vez que garantirá melhores prognósticos e eliminação da doença de forma efetiva (Rodrigues \& Domingues, 2018). No Brasil o Ministério da Saúde preconiza no mínimo 6 consultas de pré-natal. Na presente abordagem foi possível identificar que $96 \%$ das mulheres realizaram pré-natal e mais de $60 \%$ realizaram 6 ou mais consultas. No entanto, apenas $75 \%$ dos diagnósticos de sífilis na gestante foram feitos no decorrer do pré-natal e $20 \%$ dos casos diagnosticados na admissão do trabalho de parto. O pré-natal inadequado já foi identificado em outros estudos, assim como falhas na prevenção da doença (Kimball et al, 2020). No Brasil dados de inadequação já foram reportados, onde os autores observaram 19,6\% de pré-natais inadequados (Araujo et al, 2006). Assim acreditamos que a assistência de qualidade, que possibilite a rotina para o diagnóstico da sífilis e sua intervenção precoce devem ser sempre considerados junto ao acompanhamento da gestante.

Além de permitir a identificação da sífilis na gestação, o pré-natal adequado propicia o tratamento e a diminuição da transmissão vertical. O risco de desfechos desfavoráveis à criança será mínimo se a gestante receber tratamento adequado e precoce durante a gestação (Rowe et al, 2018; Kimball et al, 2020). Entretanto a intervenção adequada no tratamento da SC parece haver falhas apontadas em outros estudos, incluindo o Brasil (Bezerra et al, 2019; Vargas et al, 2018), onde o atual inquérito pode corroborar com os dados já relatados, pois $25 \%$ das mães não realizaram nenhum tratamento durante o pré-natal e $93 \%$ dos tratamentos maternos realizados foram considerados inadequados, sendo que quase metade (45\%) possuía mais de um critério para ser considerado inapropriado.

\section{Conclusão}

A prevalência descrita no estudo para SC permite concluir que a região avaliada carece de estratégias eficientes na prevenção e tratamento da doença. Além disso as associações das frequências da SC com os fatores abordados no estudo permitem provocar questionamentos sobre a qualidade equitativa da assistência pré-natal e perinatal ofertada à população. Contudo a melhora da qualidade do pré-natal e um acompanhamento rigoroso dos casos de SC são fatores a serem ponderados, a fim de diminuir os fatores de risco e as sequelas da transmissão vertical da sífilis.

Entretanto a tentativa de reconstituir a trajetória assistencial dos casos de sífilis congênita a partir de dados de um estudo transversal e da busca de informação em prontuários médicos é uma limitação deste estudo. Algumas fichas consultadas não apresentavam todas as informações relevantes para o objetivo deste estudo, além de possuírem qualidade de preenchimento variável e muitas vezes incompleta, o que torna um fator limitante à qualidade dos dados epidemiológicos, já observado em outro estudo no país. Assim, é necessário avaliar os dados com cautela e com discernimento, visto que as informações foram captadas até o instante em que a pesquisa foi realizada e caracterizam a população específica desse estudo.

Contudo novos estudos pela busca de indicadores epidemiológicos para acompanhar e avaliar as discrepâncias entre as diferentes regiões do país, bem como os tipos de serviços à saúde, público e privado serão fundamentais para viabilizar melhorias no serviço de saúde e qualidade de vida para as gestantes e os seus conceptos no que tange ao combate à sífilis 
congênita.

\section{Agradecimentos}

Os autores agradecem ao apoio e incentivo científico da pró-reitoria de pesquisa e pós-graduação da Universidade Federal do Triângulo Mineiro. WFR agradeço ao Programa Nacional de Pós Doutorado da CAPES pela concessão de bolsa de Pós-doutoramente pelo programa de Pós-graduação em Ciências da Saúde da Universidade Federal do Triângulo Mineiro.

\section{Referências}

Almeida, K. C., Lindolfo, L. C., \& Alcântara, K. C. (2009). Sífilis em gestantes atendidas em uma unidade de saúde pública de Anápolis, Goiás. Rev. bras. anal. Clin, 41(3), 181-184.

Andrade, A. L. M. B., et al. (2018). Diagnóstico tardio de sífilis congênita: uma realidade na atenção à saúde da mulher e da criança no brasil. Revista Paulista de Pediatria, 36(03), 376-381. https://doi.org/10.1590/1984-0462.

Arango, H. G. (2001). Bioestatística teórica e computacional. In: Bioestatística teórica e computacional, Rio de Janeiro: Guanabara Koogan, 235p.

Araujo, E. C., et al. (2006). Importância do pré-natal na prevenção da sífilis congênita. Revista Paraense de Medicina, 20(1), 47-51.

Avelleira, J. C. R., \& Bottino, G. (2006). Sífilis: diagnóstico, tratamento e controle. Anais brasileiros de dermatologia, 81(2), 111-126.

Bezerra, M. L. M. B. et al. (2019). Congenital syphilis as a measure of maternal and child healthcare, Brazil. Emerging infectious diseases, 25(8), 1469-1476. https://doi.org/10.3201/eid2508.180298

Cooper, J. M., \& Sánchez, P. J. (2018). Congenital syphilis. Semin Perinatol, 42(3), 176-184. 10.1053/j.semperi.2018.02.005.

De Lorenzi, D. R. S., \& Madi, J. M. (2001). Sífilis congênita como indicador de assistência pré-natal. Revista Brasileira de Ginecologia e Obstetrícia, 23(10), 647-652. https://doi.org/10.1590/S0100-72032001001000006.

Domingues, R. M., et al. (2013). Congenital syphilis: a sentinel event in antenatal care quality. Rev Saude Publica, 47(1), 147-56. 10.1590/s003489102013000100019

Forrestel, A. K., Kovarik, C. L., \& Katz, K. A. (2020). Sexually acquired syphilis: Laboratory diagnosis, management, and prevention. J Am Acad Dermatol, 82(1), 17-28. 10.1016/j.jaad.2019.02.074.

Galvis, A. E., \& Arrieta, A. (2020). Congenital Syphilis: A U.S. Perspective. Children (Basel), 7(11), 203. 10.3390/children7110203.

Kimball, A., et al. (2020). Missed Opportunities for Prevention of Congenital Syphilis - United States, 2018. MMWR Morb Mortal Wkly Rep, 69(22), 661665. 10.15585/mmwr.mm6922a1.

Koundanya, V. V., \& Tripathy, K. (2021). Syphilis Ocular Manifestations. 2021 Feb 14. In: StatPearls. Treasure Island (FL): StatPearls Publishing.

Kroeger, K. A., et al. (2018). Pathways to Congenital Syphilis Prevention: A Rapid Qualitative Assessment of Barriers, and the Public Health Response, in Caddo Parish, Louisiana. Sex Transm Dis, 45(7), 442-446. 10.1097/OLQ.0000000000000787.

Lafetá, K. R. G. et al. (2016). Sífilis materna e congênita, subnotificação e difícil controle. Revista Brasileira de Epidemiologia, 19(01), 63-74. https://doi.org/10.1590/1980-5497201600010006.

Magalhães, D. M. S., et al. (2013). Sífilis materna e congênita: ainda um desafio. Cadernos de Saúde Pública, 29(6), 1109-1120. https://doi.org/10.1590/S0102-311X2013000600008.

Monu, A. M. et al. (2020). Epidemiological aspects of syphilis. Medical-Surgical Journal, 24(2), 307-312.

Paiva, K. M., et al. (2020). Perfil epidemiológico da sífilis materna e congênita em florianópolis, 2016-2017. Brazilian Journal of Development, 6(8), 5475054760. https://doi.org/10.34117/bjdv6n8-042.

Phiske, M. M. (2014). Current trends in congenital syphilis. Indian J Sex Transm Dis AIDS, 35(1), 12-20. 10.4103/0253-7184.132404.

Rodrigues, D. C., \& Domingues, R. M. S. M. (2018). Management of syphilis in pregnancy: Knowledge and practices of health care providers and barriers to the control of disease in Teresina, Brazil. Int J Health Plann Manage, 33(2), 329-344. 10.1002/hpm.2463.

Rodrigues, W. F., et al. (2016). Antibiotic Resistance of Bacteria Involved in Urinary Infections in Brazil: A Cross-Sectional and Retrospective Study. Int J Environ Res Public Health, 13(9), 918. 10.3390/ijerph13090918.

Rose, V. L. (1998). CDC releases the 1998 Guidelines for the Treatment of Sexually Transmitted Diseases. Am Fam Physician, 15;57(8), 2003-4, 2007-8.

Rowe, C. R., Newberry, D. M., \& Jnah, A. J. (2018). Congenital Syphilis: A Discussion of Epidemiology, Diagnosis, Management, and Nurses' Role in Early Identification and Treatment. Adv Neonatal Care, 18(6), 438-445. 10.1097/ANC.0000000000000534. 
Research, Society and Development, v. 11, n. 1, e54511125419, 2022

(CC BY 4.0) | ISSN 2525-3409 | DOI: http://dx.doi.org/10.33448/rsd-v11i1.25419

Santos, S. B., et al. (2019). Acquired Syphilis: construction and validation of educational technology for adolescents. Journal of Human Growth and Development, 29(1), 65-74. http://dx.doi.org/10.7322/jhgd.157752.

Vargas, L., et al. (2018). High prevalence of syphilis in parturient women and congenital syphilis cases in public maternities in Salvador-Bahia, Brazil. BJOG, 125(10), 1212-1214. 10.1111/1471-0528.15304.

Woods, C. R. (2005). Syphilis in children: congenital and acquired. Semin Pediatr Infect Dis, 16(4), 245-57. 10.1053/j.spid.2005.06.005. 